\title{
External validation of a low HEAR score to identify emergency department chest pain patients at very low risk of major adverse cardiac events without troponin testing
}

\author{
Connor M. O'Rielly ${ }^{1} \cdot$ James E. Andruchow ${ }^{1} \cdot$ Andrew D. McRae $^{1}$ (D)
}

Received: 3 January 2021 / Accepted: 5 June 2021 / Published online: 17 July 2021

(c) The Author(s), under exclusive licence to Canadian Association of Emergency Physicians (CAEP)/ Association Canadienne de Médecine d'Urgence (ACMU) 2021

\begin{abstract}
Background The history, ECG, age, risk factor (HEAR) score has been proposed to identify patients at sufficiently low risk of acute coronary syndrome that they may not require troponin testing. The objective of this study was to externally validate a low HEAR score to identify emergency department (ED) patients with chest pain at very low risk of 30-day major adverse cardiac events (MACE).

Methods This was a secondary analysis of a prospective cohort of patients requiring troponin testing to rule out myocardial infarction (MI) in a large urban ED. HEAR scores were calculated in two cohorts: (1) patients with no known history of coronary artery disease (CAD); and (2) all eligible patients. The proportion of patients classified as very low risk, sensitivity, specificity, predictive values and likelihood ratios at each cut-off were quantified for index acute myocardial infarction (AMI) and 30-day MACE at HEAR $=0$ and HEAR $\leq 1$ thresholds.

Results Of the 1150 patients included in this study, 820 (71.3\%) had no history of CAD, 97 (8.4\%) had index AMI and $123(10.7 \%)$ had 30-day MACE. In patients with no prior history of CAD, HEAR $\leq 1$ identified $202(24.6 \%)$ of patients as very low risk for 30-day MACE with 98.4\% (95\% CI 91.6-99.9\%) sensitivity. Among all patients, HEAR $\leq 1$ identified 202 (17.6\%) patients as very low risk for 30-day MACE with 99.2\% (95\% CI 95.6-99.9\%) sensitivity.

Conclusions A HEAR score $\leq 1$ can identify more than $17 \%$ of all patients as very low risk for index AMI and 30-day MACE and unlikely to benefit from troponin testing. Broad implementation of this strategy could lead to significant resource savings.
\end{abstract}

Keywords HEAR score $\cdot$ Chest pain $\cdot$ Risk stratification

\section{Résumé}

Contexte Le score HEAR (History, ECG, Age, Risk Factor) a été proposé pour identifier les patients présentant un risque suffisamment faible de syndrome coronarien aigu pour ne pas nécessiter de test à la troponine. L'objectif de cette étude était de valider à l'externe un faible score HEAR afin d'identifier les patients du service d'urgence (DE) souffrant de douleurs thoraciques à très faible risque d'événements cardiaques indésirables majeurs (MACE) de 30 jours.

Méthodes Il s'agissait d'une analyse secondaire d'une cohorte prospective de patients nécessitant un test à la troponine pour exclure un infarctus du myocarde (IM) dans un grand service d'urgence urbain. Les scores HEAR ont été calculés dans deux cohortes : (1) les patients sans antécédents connus de maladie coronarienne (MC); et (2) tous les patients éligibles. La proportion de patients classés à très faible risque, la sensibilité, la spécificité, les valeurs prédictives et les rapports de vraisemblance à chaque seuil ont été quantifiés pour l'infarctus aigu du myocarde (IAM) de l'index et la MACE à 30 jours aux seuils HEAR $=0$ et $\operatorname{HEAR} \leq 1$.

Andrew D. McRae

amcrae@ucalgary.ca

1 Department of Emergency Medicine, Cumming School of Medicine, University of Calgary, 3280 Hospital Drive NW, Room 3E34, Calgary, AB T2N 4Z6, Canada 
Résultats Sur les 1150 patients inclus dans cette étude, 820 (71.3\%) n'avaient pas d'antécédents de coronaropathie, 97 (8.4\%) avaient un IAM index et $123(10.7 \%)$ avaient une MACE à 30 jours. Chez les patients sans antécédents de coronaropathie, HEAR $\leq 1$ a identifié $202(24,6 \%)$ des patients comme présentant un très faible risque de MACE à 30 jours avec une sensibilité de 98.4\% (IC 95\% 91.6-99.9\%). Parmi tous les patients, HEAR $\leq 1$ a identifié 202 (17.6\%) patients comme présentant un très faible risque de MACE à 30 jours avec une sensibilité de 99.2\% (IC 95\% 95.6-99.9\%).

Conclusions Un score HEAR $\leq 1$ peut identifier plus de $17 \%$ de tous les patients comme étant à très faible risque d'IAM d'index et de MACE à 30 jours et peu susceptibles de bénéficier d'un test à la troponine. La mise en œuvre à grande envergure de cette stratégie pourrait permettre de réaliser d'importantes économies de ressources.

\section{Clinician's Capsule}

What is known about the topic?

A low History, ECG, Age and Risk Factor (HEAR) score may rule out 30-day major adverse cardiac events (MACE).

\section{What did the study ask?}

Can a very low HEAR score rule-out 30-day MACE for ED chest pain patients without utilizing troponin testing?

\section{What did this study find?}

A HEAR Score $\leq 1$ can identify $>17 \%$ of patients as very low risk for 30-day MACE with sensitivity over $99 \%$.

\section{Why does this study matter to clinicians?}

Identifying very low risk chest pain patients unlikely to benefit from troponin testing may lead to significant healthcare resource savings.

\section{Background}

Chest pain and symptoms of acute coronary syndromes are a leading cause of emergency department (ED) visit worldwide, leading more than 500,000 Canadians to present to the ED annually [1]. Identifying the $10-15 \%$ of those patients actually experiencing acute coronary syndromes requires a thorough diagnostic evaluation, often with serial biomarker testing over hours, and contributes to increased ED length of stay and resource utilization [2]. Consequently, strategies to identify ED patients at low risk of acute coronary syndromes and suitable for early discharge are of significant interest. Most of these clinical risk prediction tools incorporate patient clinical characteristics and cardiac biomarkers [3, 4], such as the widely used History, Electrocardiogram, Age, Risk Factors and Troponin (HEART) score [5]. The HEART score was developed in 2008 as a tool to rapidly risk stratify undifferentiated ED patients with possible acute coronary syndromes using readily available clinical criteria and

assigns a score of 0,1 or 2 for each of the five clinical features composing the HEART acronym. A HEART score of less than or equal to 3 identifies chest pain patients at low risk of poor short-term outcomes, a finding that has since been reaffirmed in numerous validation studies and a meta-analysis [6-10].

Recently, Smith et al. proposed a modified version of the original HEART score, omitting the troponin component in an attempt to identify patients at such low risk of 30-day major adverse cardiac events that they may not require any biomarker testing at all (Appendix A, Table 4) [11]. In a secondary analysis of data from the HEART Pathway Implementation study $[4,12]$ they tested the prognostic ability of very low HEAR scores in over 4,000 patients with symptoms of possible acute coronary syndromes and no known history of coronary artery disease. They found that a HEAR score $\leq 1$ occurred in $9.0 \%$ and yielded a sensitivity of $97.8 \%$ (95\% CI 94.5-99.4\%) for 30-day major adverse cardiac events, suggesting that troponin testing is of low utility for this population [11]. Several studies have attempted to validate this strategy with promising results $[13,14]$.

If broadly validated, this strategy could substantially reduce costs and ED length of stay compared to current approaches that involve serial troponin testing over several hours for many patients. While some patients may have acute myocardial infarction ruled out using a single high-sensitivity troponin measurement, most require serial troponin measurements. The ability to rule out acute myocardial infarction and 30-day major adverse cardiac events using only clinical criteria obviates the need for both initial and serial troponin testing, leading to substantial reductions in length of stay. The objective of this investigation was to externally validate in a Canadian population the ability of a very low HEAR score to identify ED patients with chest pain who are at very low risk of index myocardial infarction and 30-day major adverse cardiac events and are unlikely to benefit from troponin testing. 


\section{Methods}

This study is a secondary analysis of data from a completed prospective cohort study that validated rapid diagnostic algorithms for myocardial infarction using high-sensitivity cardiac troponin-T $[15,16]$ at a large urban level one trauma and regional percutaneous coronary intervention centre in Calgary, AB from August 2014 to September 2016. The detailed methods of the parent study are described elsewhere $[15,16]$. In brief, trained research assistants approached patients' attending ED physicians who prospectively collected clinical variables including symptoms, cardiovascular risk factors, medical history, physicians' assessment of the probability of acute coronary syndromes (low, moderate, high risk) and HEART score. ECG and laboratory data, including serial high-sensitivity cardiac troponin $\mathrm{T}$ results were obtained from hospital records. Outcomes were obtained from a detailed review of hospital administrative data, electronic records and a provincial cardiac outcome registry [17], along with 30-day telephone follow-up. This study was conducted in alignment with the Transparent Reporting of a multivariable prediction model for Individual Prognosis or Diagnosis (TRIPOD) statement [18] and an associated TRIPOD checklist can be found in the supplementary materials (Appendix B). Both this analysis and the original cohort study were approved by the University of Calgary Conjoint Health Research Ethics Board.

\section{Population}

The study population included patients aged $\geq 25$ years presenting to the ED with chest pain and requiring troponin testing to rule out myocardial infarction per the attending ED physician. Patients with ST-elevation myocardial infarction, clear acute ischemic changes or new arrhythmia on ECG, hemodynamic instability, advanced renal failure, a diagnosis of acute coronary syndrome in the 30 days preceding the visit, or a failure to obtain consent were excluded. We used two a priori-defined cohorts in our analysis. First, to create a similar population to the HEAR score derivation by Smith et al., we included only patients without known coronary artery disease, as determined by the patients' attending physician. Second, to verify generalizability among all patients presenting to the ED with chest pain, we included all patients, including those with a prior history of coronary artery disease.

\section{Outcomes}

The primary outcome was index myocardial infarction (diagnosed within $24 \mathrm{~h}$ of ED arrival at the index encounter) as defined by the 3 rd Universal definition of myocardial infarction [19], including the rise and/or fall of high sensitivity cardiac troponin with at least one value above the 99th percentile. The co-primary outcome was 30-day major adverse cardiac events; a composite endpoint of myocardial infarction, cardiac death and urgent revascularization (nonelective coronary artery bypass grafting or percutaneous coronary intervention. All outcomes were independently adjudicated by two board-certified physicians with disagreements resolved by consensus.

\section{Statistical analysis}

HEAR scores were calculated for all patients. Listwise deletion was used to remove patients with missing data for any of the HEAR score variables from the analysis. The efficiency and diagnostic performance of HEAR $=0$ and $\leq 1$ was quantified using the proportion of patients identified as very low risk for 30-day major adverse cardiac events and index myocardial infarction as well as sensitivity, negative predictive value, and negative likelihood ratio ( - LR) for very low-risk patients, and specificity, positive predictive value (PPV) and positive likelihood ratio (+LR) amongst those not labelled as very low risk. This analysis was performed first in patients with no known history of coronary disease, then among all included patients (without and with a history of coronary artery disease).

Descriptive statistics were tabulated for patient characteristics and presented as means (SD) and proportions. Major adverse cardiac events and its components of acute myocardial infarction, revascularization and death are presented as $n(\%)$, such that a patient who experiences acute myocardial infarction and subsequent revascularization is represented as 1 30-day major adverse cardica event in any tables. All measures of prognostic and diagnostic performance are presented in tabular form with exact $95 \%$ confidence intervals for binomial proportions. All statistical analyses were completed using R Version 4.0.0 (www.r-project.org) equipped with the 'dplyr' package [20].

\section{Results}

A total of 1,167 eligible patients with at least one hs-cTnT sample were included in the parent study. In all, 17 patients were excluded due to missing HEAR score data leaving a total of 1,150 patients eligible for the predefined analyses. For the first analysis looking only at patients without known coronary artery disease, 330 patients with known coronary artery disease were excluded leaving a total of 820 patients. The cohort for the second analysis re-integrated all patients with known coronary artery disease, thus utilizing the entire cohort of 1,150 patients. The original study had complete 
Table 1 Baseline characteristics of patients with and without known coronary artery disease

\begin{tabular}{lcc}
\hline Characteristic & $\begin{array}{l}\text { Patients without } \\
\text { known CAD }(n= \\
820)\end{array}$ & $\begin{array}{l}\text { Patients with } \\
\text { known CAD }(n= \\
330)\end{array}$ \\
\hline Patient demographics & & \\
Mean age (SD) & $57.5(14.5)$ & $66.2(12.7)$ \\
Female sex (\%) & $391(47.7)$ & $97(29.4)$ \\
Risk factors & & \\
Hypertension (\%) & $357(43.5)$ & $257(77.9)$ \\
Diabetes (\%) & $85(10.4)$ & $95(28.8)$ \\
Hyperlipidemia (\%) & $246(30.0)$ & $231(70.0)$ \\
Family history (\%) & $151(18.4)$ & $73(22.1)$ \\
Smoker (\%) & $114(13.9)$ & $45(13.6)$ \\
Obesity (\%) & $160(19.5)$ & $78(23.6)$ \\
HEAR scores & & $0(0)$ \\
0 & $65(7.9)$ & $0(0)$ \\
1 & $137(16.7)$ & $23(6.9)$ \\
2 & $184(22.4)$ & $67(20.3)$ \\
3 & $200(24.4)$ & $82(24.8)$ \\
4 & $127(15.5)$ & $88(26.7)$ \\
5 & $76(9.3)$ & $55(16.7)$ \\
6 & $22(2.7)$ & $15(4.5)$ \\
7 & $7(0.9)$ & $0(0)$ \\
8 & $2(0.2)$ & $38(11.8)$ \\
30 -day outcomes & $57(7.0)$ & $3(0.9)$ \\
MI (\%) & $1(0.1)$ & \\
Revascularization $(\%)$ & & \\
Death (\%) & & \\
\hline
\end{tabular}

Results are presented as $n(\%)$. It is possible for the same patient to experience more than one of the listed outcomes and each individual event is counted in this table

$C A D$, coronary artery disease; HEAR, History ECG Age Risk Factors; $S D$, standard deviation; $M I$, myocardial infarction

follow-up for all patients. The participant flow diagram is shown in Appendix C, Fig. 1.

Among the 820 patients without known coronary artery disease, 57 (7.0\%) and 64 (7.8\%) patients experienced index myocardial infarction and 30-day major adverse cardiac events, respectively. A HEAR score was calculated for all patients with $65(7.9 \%)$ having a HEAR $=0,137(16.7 \%)$ with a HEAR $=1$ and the remaining $618(75.4 \%)$ patients having HEAR $>1$. Characteristics and outcome information for patients in both analyses are shown in Table 1.

None of the 65 patients $(7.9 \%)$ with HEAR $=0$ experienced either of the co-primary outcomes, yielding sensitivities of $100 \%$ (95\% CI 93.7-100\%) and $100 \%$ (95\% CI 94.4-100\%) for myocardial infarction and 30-day major adverse cardiac events, respectively. The specificity of this strategy was low for both clinical endpoints, at $8.5 \%$ (95\% CI $6.6-10.7 \%)$ for index myocardial infarction and 8.6\% (95\%
CI 6.7-10.8\%) for 30-day major adverse cardiac events. Of the 202 patients $(24.6 \%)$ with a HEAR score $\leq 1$, one experienced an index myocardial infarction and underwent subsequent revascularization, yielding sensitivities of $98.3 \%$ (95\% CI 90.6-99.9\%) for index myocardial infarction and 98.4\% (95\% CI 91.6-99.9\%) for 30-day major adverse cardiac events. These high sensitivities coincided with specificities of $26.3 \%$ (95\% CI 23.3-29.6\%) for index myocardial infarction and $26.6 \%$ (95\% CI 23.5-29.9\%) for 30-day major adverse cardiac events. The negative predictive value of this strategy was high at $99.5 \%$ (95\% CI 96.6-99.9\%) for both clinical endpoints. All classification characteristics for this primary analysis are shown in Table 2.

The second analysis was performed with 1,150 patients, including the 320 patients with known coronary artery disease previously excluded from the first analysis. Again, none of the patients with HEAR $=0$ experienced either of the co-primary clinical endpoints, while the same single patient with HEAR $=1$ accounted for both an index acute myocardial infarction and subsequent revascularization (i.e., major adverse cardiac events) at 30-days. None of the added patients had a HEAR score below 2 given that having known coronary artery disease garners an automatic 'Risk Factor' HEAR component score of 2. While the proportion of patients identified as 'very low risk' by both HEAR $=0$ and $\leq 1$ did decrease secondary to an increased denominator, sensitivity for both the index myocardial infarction and 30-day major adverse cardiac events outcomes remained above $98.9 \%$ (95\% CI 94.3-99.9\%). Specifically, the HEAR $\leq 1$ strategy identified $17.6 \%$ of patients as low risk and yielded sensitivities of 98.9\% (95\% CI 94.3-99.9\%) and 99.2\% (95\% CI 95.6-99.9\%) for index myocardial infarction and 30-day major adverse cardiac events, respectively. Compared to the first analysis, the specificity of the HEAR $\leq 1$ cutoff was lower: $19.1 \%$ (95\% CI 16.7-21.6\%) for index myocardial infarction and $19.6 \%$ (95\% CI $17.2-22.1 \%$ ) for 30 -day major adverse cardiac events. The negative predictive value of the HEAR $\leq 1$ strategy was unchanged by the addition of patients with known coronary artery disease remaining high at $99.5 \%$ (95\% CI 96.6-99.9\%). All other classification characteristics for the second analysis are shown in Table 3.

\section{Discussion}

This study sought to validate the utility of a very low HEAR score in identifying patients at very low risk of 30-day major adverse cardiac events and index myocardial infarction. In our study, a HEAR $\leq 1$ cut-off identified $24.6 \%$ of chest pain patients without known coronary artery disease as low risk for both outcomes with sensitivities above $98 \%$ and negative predictive values $>99.5 \%$. When including patients with 
Table 2 Test characteristics of HEAR scores 0 and $\leq 1$ for the detection of myocardial infarction at the index visit and major adverse cardiac events at 30 days among patients with no known coronary artery disease $(n=820)$
Table 3 Test characteristics of HEAR scores 0 and $\leq 1$ for the detection of major adverse cardiac events at 30 days and myocardial infarction at the index visit among all patients, including those with known coronary artery disease $(n=$ 1150)

\begin{tabular}{lll}
\hline Risk score & $\begin{array}{l}\text { HEAR 0 (95\% CI) } \\
n=65\end{array}$ & $\begin{array}{l}\text { HEAR } \leq 1 \text { (95\% CI) } \\
n=202\end{array}$ \\
\hline $\begin{array}{l}\text { Proportion of all patients Low risk (\%) } \\
\text { Index MI }\end{array}$ & $7.9(6.2-9.9)$ & $24.6(21.7-27.7)$ \\
Number of missed events & 0 & 1 \\
Sensitivity & $100(93.7-100)$ & $98.3(90.6-99.9)$ \\
Specificity & $8.5(6.6-10.7)$ & $26.3(23.3-29.6)$ \\
PPV & $7.6(7.4-7.7)$ & $9.1(8.6-9.5)$ \\
NPV & $100(-)$ & $99.5(96.6-99.9)$ \\
+ LR & $1.1(1.1-1.1)$ & $1.3(1.3-1.4)$ \\
- LR & - & $0.1(0.0-0.5)$ \\
30-day MACE & & 1 \\
Number of missed events & 0 & $98.4(91.6-99.9)$ \\
Sensitivity & $100(94.4-100)$ & $26.6(23.5-29.9)$ \\
Specificity & $8.6(6.7-10.8)$ & $10.2(9.7-10.7)$ \\
PPV & $8.5(8.3-8.7)$ & $99.5(96.6-99.9)$ \\
NPV & $100(-)$ & $1.3(1.3-1.4)$ \\
+ LR & $1.1(1.1-1.1)$ & $0.1(0.0-0.4)$ \\
- LR & - & \\
\hline
\end{tabular}

The same patient with a HEAR $\leq 1$ experienced index $\mathrm{MI}$ and a subsequent 30-day revascularization

$H E A R$, History ECG Age Risk Factors; $M A C E$, major adverse cardiac events; $P P V$, positive predictive value; $N P V$, negative predictive value; $+L R$, positive likelihood ratio; $-L R$, negative likelihood ratio; $M I$, myocardial infarction

\begin{tabular}{lll}
\hline Risk score & $\begin{array}{l}\text { HEAR 0 (95\% CI) } \\
n=65\end{array}$ & $\begin{array}{l}\text { HEAR } \leq 1 \text { (95\% CI) } \\
n=202\end{array}$ \\
\hline $\begin{array}{l}\text { Proportion of all patients low risk (\%) } \\
\text { Index MI }\end{array}$ & $5.7(4.4-7.1)$ & $17.6(15.4-19.9)$ \\
Number of missed events & 0 & 1 \\
Sensitivity & $100(96.2-100)$ & $98.9(94.3-99.9)$ \\
Specificity & $6.2(4.8-7.8)$ & $19.1(16.7-21.6)$ \\
PPV & $8.8(8.6-8.9)$ & $9.9(9.6-10.3)$ \\
NPV & $100(-)$ & $99.5(96.6-99.9)$ \\
+ LR & $1.1(1.1-1.1)$ & $1.2(1.2-1.3)$ \\
- LR & - & $0.1(0.1-0.4)$ \\
30-day MACE & & 1 \\
Number of Missed Events & 0 & $99.2(95.6-99.9)$ \\
Sensitivity & $100(97.1-100)$ & $19.6(17.2-22.1)$ \\
Specificity & $6.3(4.9-8.0)$ & $12.9(12.5-13.3)$ \\
PPV & $11.3(11.2-11.5)$ & $99.5(96.6-99.9)$ \\
NPV & $100(-)$ & $1.2(1.2-1.3)$ \\
+ LR & $1.1(1.1-1.1)$ & $0.0(0.0-0.3)$ \\
- LR & - &
\end{tabular}

The same patient with a HEAR $\leq 1$ experienced index MI and a subsequent 30-day revascularization

$H E A R$, History ECG Age Risk Factors; $M A C E$, major adverse cardiac events; $P P V$, positive predictive value; $N P V$, negative predictive value; $+L R$, positive likelihood ratio; $-L R$, negative likelihood ratio; $M I$, myocardial infarction known coronary artery disease the proportion identified as low risk decreased to $17.6 \%$, but sensitivity and negative predictive value for both index myocardial infarction and 30-day major adverse cardiac events remained above $98.9 \%$ and $99.5 \%$, respectively. These results suggest that a HEAR score $\leq 1$ may be sufficient to safely identify nearly 
a quarter of patients without known coronary artery disease and over $17 \%$ of all patients as very low risk for 30-day major adverse cardiac events and unlikely to benefit from biomarker testing.

The proportion of patients identified as very low risk by a HEAR score $\leq 1$ was nearly three times higher in our study compared to the original manuscript by Smith et al. [5] (24.6\% vs. 9.0\%). We suspect that, despite a higher mean age in our cohort, this difference is owing to the lower prevalence of known coronary artery disease risk factors in our study including obesity ( $20.7 \%$ vs. $31.1 \%)$, and smoking $(13.9 \%$ vs. $48.8 \%)$. While the original study had more HEAR $\leq 1$ patients with these risk factors, a HEAR score $\leq 1$ requires that each patient has a maximum of only two of the listed risk factors. Consequently, despite a higher prevalence of these risk factors across their cohort, risks likely remained low at the individual level which may explain the nearly equivalent incidence of 30-day major adverse cardiac events ( $0.5 \%$ vs. $0.9 \%)$ and sensitivities for this outcome ( $98.4 \%$ vs. $97.8 \%$ ) between our analysis and that of Smith et al.

The clinical utility of the HEAR score in the derivation study was somewhat limited by the decision to exclude patients with known coronary artery disease. This is an understandable decision given that due to the scoring system of the HEAR score, an individual with known coronary artery disease will automatically earn a minimum score of 2 thus ensuring that they do not qualify for the strategy validated herein. However, excluding them entirely from the analysis creates a pre-screened population of patients that is no longer representative of the broader chest pain population seen in an ED practice. Given this, a treating ED clinician would have to consider the patient's coronary artery disease status before even beginning the chest pain assessment, adding to cognitive load. Excluding individuals with known coronary artery disease also decreases the denominator on which the proportion of patients that are discharge eligible is calculated and is likely to inflate estimates of the proportion of patients classified as low-risk. By including these patients in our second analysis we demonstrated that the safety of the strategy is unchanged and were able to obtain an estimate of the proportion that can be identified as a low risk that is more reflective of a true ED chest pain population (17.6\% versus $24.6 \%$ ). This more representative value is closely aligned with recent evidence from a study of administrative ED data [13] that found that 19\% of ED patients with and without coronary artery disease had a HEAR $\leq 1$ score and were unlikely to benefit from biomarker testing.

There has been continuous advancement in the sensitivity and precision of cardiac troponin biomarkers which has facilitated a reduction in the time required to safely rule out acute myocardial infarction in the ED. With previous generations of troponin, serial sampling was almost always required; more recently, a number of $0 / 2 \mathrm{~h}, 0 / 1 \mathrm{~h}$, and single measure troponin strategies have emerged with high sensitivity for excluding the diagnosis of acute myocardial infarction [21, 22]. This, of course, contributes to immediate cost savings by reducing the net number of troponin tests performed. However, only a small proportion of patients can have myocardial infarction ruled out with a single troponin measurement, and most require serial measurement. Herein lies the greatest potential contribution of the very low HEAR score strategy that we have validated: the ability to rule out acute myocardial infarction in a sizeable amount of chest pain patients using only clinical criteria and eliminating the need for any single or serial troponin measurement in very low-risk patients.

Our study may be limited by the exclusion of patients with clear ECG ischemia such that it may not apply to all ED patients with chest pain. However, clear ischemic ECG changes would have resulted in a HEAR score of 2 points or higher, which - if anything - make our estimates of sensitivity more conservative. Patients who experienced ST-elevated myocardial infarction were also excluded because they are not typically risk stratified using tools such as a HEAR score but are instead put on a rapid trajectory to coronary intervention. Smith et al. used a net reclassification index (NRI) to assess the incremental value of adding a troponin measurement for distinguishing low from high-risk patients [23]. They determined that the addition of a single troponin to a HEAR score of $\leq 1$ did not significantly improve risk classification [11]. We did not perform this analysis because only one patient with HEAR $\leq 1$ had a 30-day major adverse cardiac event, suggesting minimal incremental benefit of highsensitivity troponin measurement in patients with very low HEAR scores.

\section{Conclusion}

In conclusion, a HEAR score $\leq 1$ can identify about onequarter of ED chest pain patients without known coronary artery disease and over $17 \%$ of patients of all ED chest pain patients as very low risk for 30-day major adverse cardiac events, and unlikely to benefit from troponin testing. Additional validation in larger prospectively collected datasets is likely required before broad clinical adoption. But this strategy, if broadly implemented, could contribute to significant resource savings, reductions in ED length of stay by eliminating the need for serial troponin testing for very low-risk patients.

Supplementary Information The online version contains supplementary material available at https://doi.org/10.1007/s43678-021-00159-y.

Funding The parent study was funded by an investigator initiated, nonrestricted research grant from Roche Diagnostics Canada. 


\section{Declarations}

Conflict of interest All authors declare that they have no conflict of interest.

\section{References}

1. Ko DT, Dattani ND, Austin PC, et al. Emergency department volume and outcomes for patients after chest pain assessment. Circ Cardiovasc Qual Outcomes. 2018;11:e004683. https://doi. org/10.1161/circoutcomes.118.004683.

2. Hess EP, Brison RJ, Perry JJ, et al. Development of a clinical prediction rule for 30-day cardiac events in emergency department patients with chest pain and possible acute coronary syndrome. Ann of Emerg Med. 2012;59:115-125.e111. https://doi.org/10. 1016/j.annemergmed.2011.07.026.

3. Liu N, Ng JCJ, Ting CEA, et al. Clinical scores for risk stratification of chest pain patients in the emergency department: an updated systematic review. J Emerg Crit Care Med. 2018. https:// doi.org/10.21037/jeccm.2018.01.10.

4. Mahler SA, Lenoir KM, Wells BJ, et al. Safely identifying emergency department patients with acute chest pain for early discharge. Circulation. 2018;138:2456-68. https://doi.org/10.1161/ CIRCULATIONAHA.118.036528.

5. Six A, Backus B, Kelder J. Chest pain in the emergency room: value of the HEART score. Neth Heart J. 2008;16:191-6. https:// doi.org/10.1007/BF03086144.

6. Laureano-Phillips J, Robinson RD, Aryal S, et al. HEART score risk stratification of low-risk chest pain patients in the emergency department: a systematic review and meta-analysis. Ann Emerg Med. 2019. https://doi.org/10.1016/j.annemergmed.2018.12.010.

7. Backus BE, Six AJ, Cullen L, et al. The HEART score for chest pain patients at the emergency department validated in a multi centre Asia-Pacific population. Eur Heart J. 2012;1:6-7. https:// doi.org/10.1093/eurheartj/ehs281.

8. Brady W, de Souza K. The HEART score: a guide to its application in the emergency department. Tur J Emerg Med. 2018;18:4751. https://doi.org/10.1016/j.tjem.2018.04.004.

9. Stopyra JP, Riley RF, Hiestand BC, et al. The HEART pathway randomized controlled trial one-year outcomes. Acad Emerg Med. 2019;26:41-50. https://doi.org/10.1111/acem.13504.

10. Streitz MJ, Oliver JJ, Hyams JM, et al. A retrospective external validation study of the HEART score among patients presenting to the emergency department with chest pain. Int Emerg Med. 2018;13:727-48. https://doi.org/10.1007/s11739-017-1743-4.

11. Smith LM, Ashburn NP, Snavely AC, et al. Identification of very low-risk acute chest pain patients without troponin testing. Emerg Med J. 2020. https://doi.org/10.1136/emermed-2020-209698 (emermed-2020-209698).
12. Mahler SA, Burke GL, Duncan PW, et al. HEART pathway accelerated diagnostic protocol implementation: prospective pre-post interrupted time series design and methods. JMIR Res Prot . 2016;5: e10. https://doi.org/10.2196/resprot.4802.

13. Moumneh T, Sun BC, Baecker A, et al. Identifying patients with low-risk of acute coronary syndrome without troponin testing: VALIDATION of the HEAR score. Am J Med. 2020. https://doi. org/10.1016/j.amjmed.2020.09.021.

14. Otsuka Y, Takeda S. Validation study of the modified HEART and HEAR scores in patients with chest pain who visit the emergency department. Acute Med Surg. 2020;7:e591. https://doi.org/10. 1002/ams2.591.

15. Andruchow JE, Boyne T, Innes G, et al. Low high-sensitivity troponin thresholds identify low-risk patients with chest pain unlikely to benefit from further risk stratification. CJC Open. 2019;1:289-96. https://doi.org/10.1016/j.cjco.2019.08.002.

16. Andruchow JE, Boyne T, Seiden-Long I, et al. Prospective comparative evaluation of the European Society of Cardiology (ESC) 1-h and a 2-h rapid diagnostic algorithm for myocardial infarction using high-sensitivity troponin-T. CJEM. 2020. https://doi.org/10. 1017/cem.2020.349.

17. Ghali WA, Knudtson ML. Overview of the alberta provincial project for outcome assessment in coronary heart disease. On BEHALF of the APPROACH investigators. Can J Cardiol. 2000;16:1225-30.

18. Moons KGM, Altman DG, Reitsma JB, et al. Transparent reporting of a multivariable prediction model for individual prognosis or diagnosis (TRIPOD): explanation and elaboration. Ann Int Med. 2015;162:W1-73. https://doi.org/10.7326/M14-0698.

19. Thygesen K, Alpert JS, Jaffe AS, et al. Third universal definition of myocardial infarction. Circulation. 2012;126:2020-35. https:// doi.org/10.1161/CIR.0b013e31826e1058.

20. Wickham H, François R, Henry L, Müller K. dplyr: A grammar of data manipulation. 2020. https://dplyr.tidyverse.org/. Accessed 15 July 2021.

21. Stepinska J, Lettino M, Ahrens I, et al. Diagnosis and risk stratification of chest pain patients in the emergency department: focus on acute coronary syndromes. A position paper of the acute cardiovascular care association. Eur Heart J Acute Cardiovasc Care. 2020;9:76-89. https://doi.org/10.1177/2048872619885346.

22. Andruchow JE, Kavsak PA, McRae AD. Contemporary emergency department management of patients with chest pain: a concise review and guide for the high-sensitivity troponin Era. Can J Cardiol. 2018;34:98-108. https://doi.org/10.1016/j.cjca.2017.11. 012.

23. Pencina MJ, D'Agostino RB Sr, D’Agostino RB Jr, et al. Evaluating the added predictive ability of a new marker: from area under the ROC curve to reclassification and beyond. Stat Med. 2008;27:157-72. https://doi.org/10.1002/sim.2929. 